\title{
INTEGRABILITY OF SUPERHARMONIC FUNCTIONS ON HÖLDER DOMAINS OF THE PLANE
}

\author{
MAKOTO MASUMOTO \\ (Communicated by Clifford J. Earle, Jr.)
}

\begin{abstract}
We prove that if $D$ is a finitely connected Hölder domain of the plane, then there exists $p>0$ for which every positive superharmonic function on $D$ is $p$-integrable over $D$ with respect to two-dimensional Lebesgue measure.
\end{abstract}

\section{INTRODUCTION}

Let $D$ be a proper subdomain of the complex plane $\mathbb{C}$. We denote by $\delta_{D}(z)$ the distance between a point $z \in D$ and the boundary $\partial D$ of $D$ :

$$
\delta_{D}(z)=\inf \{|z-\zeta| \mid \zeta \in \partial D\} .
$$

The quasi-hyperbolic metric $k_{D}$ in $D$ is defined by

$$
k_{D}\left(z_{1}, z_{2}\right)=\inf _{\gamma} \int_{\gamma} \frac{|d z|}{\delta_{D}(z)}, \quad z_{1}, z_{2} \in D,
$$

where the infimum is taken over all rectifiable arcs $\gamma$ joining $z_{1}$ and $z_{2}$ in $D$ (cf. Gehring-Palka [4] and Gehring-Osgood [3]). Let $a \in D$. If there are constants $\alpha$ and $A$ such that

$$
k_{D}(a, z) \leq \alpha \log \frac{\delta_{D}(a)}{\delta_{D}(z)}+A, \quad z \in D,
$$

then $D$ is called a Hölder domain.

Denote by $S^{+}(D)$ (resp. $\left.H^{+}(D)\right)$ the set of positive superharmonic (resp. harmonic) functions on $D$ and by $L^{p}(D), 0<p<+\infty$, the spaces of Lebesgue measurable functions on $D$ which are $p$-integrable over $D$ with respect to two-dimensional Lebesgue measure. We ask whether there is $p>0$ such that $S^{+}(D) \subset L^{p}(D)$ (or $\left.H^{+}(D) \subset L^{p}(D)\right)$. It is clear that if for each $z_{0} \in D$ there is $M>0$ such that the inequality

$$
\iint_{D} u(z)^{p} d x d y \leq M u\left(z_{0}\right)^{p}, \quad z=x+i y,
$$

Received by the editors December 11, 1990 and, in revised form, July 31, 1991.

1991 Mathematics Subject Classification. Primary 31A05; Secondary 30C20.

Key words and phrases. Hölder domain, quasi-hyperbolic metric, superharmonic function, harmonic function, conformal mapping. 
holds for all $u \in S^{+}(D)$ (resp. $u \in H^{+}(D)$ ), then $S^{+}(D) \subset L^{p}(D)$ (resp. $\left.H^{+}(D) \subset L^{p}(D)\right)$. Conversely, if $S^{+}(D) \subset L^{p}(D)$ (resp. $H^{+}(D) \subset L^{p}(D)$ ), then for each $z_{0} \in D$ there is $M>0$ such that (2) holds for all $u \in S^{+}(D)$ (resp. $u \in H^{+}(D)$ ) (see Suzuki [13]).

In 1972 Armitage [1] proved that $S^{+}(D) \subset L^{p}(D)$ for $0<p<2$ if $D$ has bounded curvature. This result was generalized by Maeda and Suzuki [7] to the case of Lipschitz domains. In our previous work [9] we considered domains satisfying an interior wedge condition and improved their results. Recently, Smith and Stegenga have proved the following theorem:

Theorem A (Smith-Stegenga [11, Corollary 4]). Let $D$ be a finitely connected domain of $\mathbb{C}$. Then the following are equivalent:

(i) $D$ is a Hölder domain.

(ii) $H^{+}(D) \subset L^{p}(D)$ for some $p>0$.

The purpose of this paper is to prove the following

Theorem. If $D$ is a finitely connected Hölder domain of $\mathbb{C}$, then $S^{+}(D) \subset L^{p}(D)$ for some $p>0$.

Combining this theorem with Theorem A, we obtain the following result:

Corollary. Let $D$ be a finitely connected domain of $\mathbb{C}$. Then the following are equivalent:

(i) $D$ is a Hölder domain.

(ii) $H^{+}(D) \subset L^{p}(D)$ for some $p>0$.

(iii) $S^{+}(D) \subset L^{p}(D)$ for some $p>0$.

It is trivial that if $S^{+}(D) \subset L^{p}(D)$ for some $p>0$, then $H^{+}(D) \subset L^{p}(D)$ for the same $p$. However, it is not known whether the converse is true. By the Riesz decomposition theorem (cf. Helms [5, Theorem 6.18]) every positive superharmonic function on $D$ is uniquely decomposed into the sum of a (Green) potential and a positive harmonic function on $D$. It should be noted that if the set of potentials on $D$ is embedded into $L^{p}(D)$ for some $p>0$, then $S^{+}(D) \subset L^{p}(D)$ for the same $p$. This observation is due to Armitage [1] and Suzuki [13, Corollary 1] and will be implicitly used in the proof of our theorem.

To prove the theorem we employ a method similar to that used in [9]. In the next section we will prepare a lemma concerning the boundary behavior of conformal mappings. Using this lemma, we will prove the theorem in $\S 3$.

\section{DISTORTION OF CONFORMAL MAPPINGS}

Let $D$ be a domain of the Riemann sphere $\widehat{\mathbb{C}}$ whose complement $\widehat{\mathbb{C}} \backslash D$ consists of more than two points. The universal covering surface of $D$ is conformally equivalent to the unit disk $\Delta$. The Poincaré metric $\lambda_{D}(z)|d z|$ for $D$ is defined by

$$
\lambda_{D}(\pi(\zeta))\left|\pi^{\prime}(\zeta)\right|=1 /\left(1-|\zeta|^{2}\right), \quad \zeta \in \Delta
$$

where $\pi: \Delta \rightarrow D$ is a holomorphic universal covering map.

The following properties of the Poincaré metric are well known. 
Lemma 1. (i) If $f$ is a conformal mapping, then $\lambda_{f(D)}(f(z))\left|f^{\prime}(z)\right|=\lambda_{D}(z)$ for $z \in D$.

(ii) If $D_{1} \subset D_{2}$, then $\lambda_{D_{2}}(z) \leq \lambda_{D_{1}}(z)$ for $z \in D_{1}$.

(iii) The inequality $\lambda_{D}(z) \delta_{D}(z) \leq 1$ holds for $z \in D$.

(iv) If $D$ is a finitely connected domain such that every component of $\widehat{\mathbb{C}} \backslash D$ contains at least two points and $\infty \notin D$, then there exists $M>0$ for which $\lambda_{D}(z) \delta_{D}(z) \geq M$ for all $z \in D$.

(v) If $D$ is simply connected and contains $\infty$, then for each compact set $K$ of $\mathbb{C}$ there exists $M>0$ such that $\lambda_{D}(z) \delta_{D}(z) \geq M$ for all $z \in D \cap K$.

For a proof of Lemma 1, see, for example, Kra [6, Chapter II, Proposition 1.1] and Masumoto [8, Lemmas 2 and 3].

We denote by $h_{D}$ the hyperbolic metric on $D$ :

$$
h_{D}\left(z_{1}, z_{2}\right)=\inf _{\gamma} \int_{\gamma} \lambda_{D}(z)|d z|, \quad z_{1}, z_{2} \in D
$$

where the infimum is taken over all rectifiable arcs $\gamma$ joining $z_{1}$ and $z_{2}$ in $D$. By Lemma 1(iii) and (iv), if $D$ is a finitely connected domain such that every component of $\widehat{\mathbb{C}} \backslash D$ is a continuum and $\infty \notin D$, then there is $M>0$ for which

$$
M k_{D}\left(z_{1}, z_{2}\right) \leq h_{D}\left(z_{1}, z_{2}\right) \leq k_{D}\left(z_{1}, z_{2}\right), \quad z_{1}, z_{2} \in D
$$

A bounded domain of $\mathbb{C}$ is called regular if it is bounded by finitely many simple closed analytic curves. Modifying the arguments in Becker-Pommerenke [2], we can establish the following

Lemma 2. If $f$ is a conformal mapping of a regular domain $D_{0}$ of $\mathbb{C}$ onto a Hölder domain $D$, then there exist $M>0$ and $0<\beta<1$ such that

$$
\left|f^{\prime}(z)\right| \leq M \lambda_{D_{0}}(z)^{\beta}
$$

for all $z \in D_{0}$.

Proof. We assume that there are constants $\alpha$ and $A$ for which (1) holds. Since

$$
k_{D}(a, z) \geq \log \left(\delta_{D}(a) / \delta_{D}(z)\right)
$$

by Gehring-Palka [4, Lemma 2.1], we have $\alpha \geq 1$. It is thus sufficient to show that $\left|f^{\prime}(z)\right| \lambda_{D_{0}}(z)^{-1+1 / 2 \alpha}$ is bounded on $D_{0}$.

The conformal mapping $f$ induces a bijection between the set of components of $\partial D_{0}$ and the set of components of $\widehat{\mathbb{C}} \backslash D$. Let $C_{0}$ be a component of $\partial D_{0}$ and let $C$ be the component of $\widehat{\mathbb{C}} \backslash D$ corresponding to $C_{0}$ under the bijection. Set $D_{1}=\widehat{\mathbb{C}} \backslash C$.

Assume first that $\infty \in D_{1}$. Let $f_{1}$ be a conformal mapping of $\Delta^{*}=\widehat{\mathbb{C}} \backslash \bar{\Delta}$, the outside of the unit disk $\Delta$, onto $D_{1}$ with $f_{1}(\infty)=\infty$. Then, in view of 
Lemma 1 and (1) and (3), we see that, for $\zeta \in \Delta^{*}$ sufficiently near $\partial \Delta^{*}$,

$$
\begin{aligned}
\log \lambda_{\Delta^{*}}(\zeta) & =\log \frac{1}{|\zeta|^{2}-1} \leq \log \frac{|\zeta|+1}{|\zeta|-1}=2 h_{D_{1}}\left(f_{1}(\zeta), \infty\right) \\
& \leq 2\left\{h_{D_{1}}\left(f_{1}(\zeta), a\right)+h_{D_{1}}(a, \infty)\right\} \leq 2 h_{D}\left(f_{1}(\zeta), a\right)+2 h_{D_{1}}(a, \infty) \\
& \leq 2 k_{D}\left(f_{1}(\zeta), a\right)+2 h_{D_{1}}(a, \infty) \\
& \leq 2 \alpha \log \frac{\delta_{D}(a)}{\delta_{D}\left(f_{1}(\zeta)\right)}+2 A+2 h_{D_{1}}(a, \infty) \\
& =2 \alpha \log \frac{1}{\delta_{D_{1}}\left(f_{1}(\zeta)\right)}+M_{1} \leq 2 \alpha \log \left\{M_{2} \lambda_{D_{1}}\left(f_{1}(\zeta)\right)\right\}+M_{1} \\
& =2 \alpha \log \frac{\lambda_{\Delta^{*}}(\zeta)}{\left|f_{1}^{\prime}(\zeta)\right|}+\left(2 \alpha \log M_{2}+M_{1}\right),
\end{aligned}
$$

where $M_{1}$ and $M_{2}$ are constants. Thus there exists $M_{3}>0$ such that

$$
\left|f_{1}^{\prime}(\zeta)\right| \lambda_{\Delta^{*}}(\zeta)^{-1+1 / 2 \alpha} \leq M_{3}
$$

for $\zeta \in \Delta^{*}$ near $\partial \Delta^{*}$. Set $\varphi=f_{1}^{-1} \circ f$ and $\Delta_{0}^{*}=f_{1}^{-1}(D) \subset \Delta^{*}$. Since $\varphi$ is extended to a homeomorphism of $D_{0} \cup C_{0}$ onto $\Delta_{0}^{*} \cup \partial \Delta^{*}$ and both $C_{0}$ and $\partial \Delta^{*}$ are analytic, we can continue $\varphi$ conformally beyond $C_{0}$ by the reflection principle. In particular, $\left|\varphi^{\prime}(z)\right| \leq M_{4}$ in a neighborhood of $C_{0}$. Hence,

$$
\begin{aligned}
\left|f^{\prime}(z)\right| \lambda_{D_{0}}(z)^{-1+1 / 2 \alpha} & =\left|f_{1}^{\prime}(\varphi(z))\right|\left|\varphi^{\prime}(z)\right| \lambda_{D_{0}}(z)^{-1+1 / 2 \alpha} \\
& \leq M_{3} \lambda_{\Delta^{*}}(\varphi(z))^{1-1 / 2 \alpha}\left|\varphi^{\prime}(z)\right| \lambda_{D_{0}}(z)^{-1+1 / 2 \alpha} \\
& \leq M_{3}\left|\varphi^{\prime}(z)\right|\left(\frac{\lambda_{\Delta_{0}^{*}}(\varphi(z))}{\lambda_{D_{0}}(z)}\right)^{1-1 / 2 \alpha} \\
& =M_{3}\left|\varphi^{\prime}(z)\right|^{1 / 2 \alpha} \leq M_{3} M_{4}^{1 / 2 \alpha}
\end{aligned}
$$

for $z$ near $C_{0}$.

The case $\infty \notin D_{1}$ can be treated similarly. Let $f_{2}$ be a conformal mapping of $\Delta$ onto $D_{1}$ with $f_{2}(0)=a$. Then, for $z \in \Delta$ sufficiently near $\partial \Delta$,

$$
\begin{aligned}
\log \lambda_{\Delta}(\zeta) & \leq 2 h_{D_{1}}\left(f_{2}(\zeta), a\right) \leq 2 k_{D}\left(f_{2}(\zeta), a\right) \\
& \leq 2 \alpha \log \frac{\delta_{D}(a)}{\delta_{D}\left(f_{2}(\zeta)\right)}+2 A \leq 2 \alpha \log \frac{\lambda_{\Delta}(\zeta)}{\left|f_{2}^{\prime}(\zeta)\right|}+M_{5},
\end{aligned}
$$

which implies that

$$
\left|f_{2}^{\prime}(\zeta)\right| \lambda_{\Delta}(\zeta)^{-1+1 / 2 \alpha} \leq M_{6} .
$$

Therefore, as in the preceding case, we see that $\left|f^{\prime}(z)\right| \lambda_{D_{0}}(z)^{-1+1 / 2 \alpha}$ is bounded near $C_{0}$.

We have shown that $\left|f^{\prime}(z)\right| \lambda_{D_{0}}(z)^{-1+1 / 2 \alpha}$ is bounded near $\partial D_{0}$. Since it is continuous in $D_{0}$, it is bounded in $D_{0}$, as desired.

\section{Proof of the theorem}

In this section we give a proof of our theorem stated in the introduction. Let $D$ be a finitely connected Hölder domain of $\mathbb{C}$. Note that $D$ is bounded by Smith-Stegenga [10, Corollary $1(\mathrm{a})]$. If $\mathbb{C} \backslash D$ has an isolated point, say $\omega$, then $D \cup\{\omega\}$ is also a Hölder domain. Furthermore, every positive superharmonic 
function on $D$ is extended to a positive superharmonic function on $D \cup\{\omega\}$. We may thus assume that each component of $\mathbb{C} \backslash D$ is a continuum.

Fix $z_{0} \in D$ and consider the set $E$ of points $(p, \gamma) \in[0,+\infty) \times \mathbb{R}$ such that there exists $M>0$ for which

$$
\iint_{D} \lambda_{D}(z)^{-\gamma} u(z)^{p} d x d y \leq M u\left(z_{0}\right)^{p}
$$

for all $u \in S^{+}(D)$. (We define $+\infty^{0}=+\infty$.)

There is a regular domain $D_{0}$ of $\mathbb{C}$ which is conformally equivalent to $D$; let $f: D_{0} \rightarrow D$ be conformal. By Lemma 2 we can choose constants $0<\beta<1$ and $M_{1}>0$ such that

$$
\left|f^{\prime}(\zeta)\right| \leq M_{1} \lambda_{D_{0}}(\zeta)^{\beta}
$$

for $\zeta \in D_{0}$. Let $G_{D}(\cdot, w)$ denote the Green's function for $D$ with pole at $w$. If $\gamma>(2 \beta-1) /(1-\beta)$, then $\gamma-\beta(\gamma+2)>-1$; hence there is $M_{2}>0$ such that

$$
\begin{aligned}
& \iint_{D} \lambda_{D}(z)^{-\gamma} G_{D}(z, w) d x d y \\
& \quad=\iint_{D_{0}} \lambda_{D_{0}}(\zeta)^{-\gamma} G_{D_{0}}\left(\zeta, f^{-1}(w)\right)\left|f^{\prime}(\zeta)\right|^{\gamma+2} d \xi d \eta \\
& \quad \leq M_{1}^{\gamma+2} \iint_{D_{0}} \lambda_{D_{0}}(\zeta)^{-\gamma+\beta(\gamma+2)} G_{D_{0}}\left(\zeta, f^{-1}(w)\right) d \xi d \eta \\
& \quad \leq M_{2} G_{D_{0}}\left(f^{-1}\left(z_{0}\right), f^{-1}(w)\right)=M_{2} G_{D}\left(z_{0}, w\right)
\end{aligned}
$$

for all $w \in D$ (cf. [9, Lemma 4]). Thus, for the (Green) potential $u$ of a positive measure $\mu$ on $D$, we have

$$
\begin{aligned}
\iint_{D} \lambda_{D}(z)^{-\gamma} u(z) d x d y & =\iint_{D}\left(\int_{D} G_{D}(z, w) d \mu(w)\right) \lambda_{D}(z)^{-\gamma} d x d y \\
& =\iint_{D}\left(\iint_{D} G_{D}(z, w) \lambda_{D}(z)^{-\gamma} d x d y\right) d \mu(w) \\
& \leq \int_{D} M_{2} G_{D}\left(z_{0}, w\right) d \mu(w)=M_{2} u\left(z_{0}\right)
\end{aligned}
$$

We have shown that (5) holds for all potentials $u$ on $D$ with $M=M_{2}$ and $p=1$. Since every positive superharmonic function on $D$ is approximated by an increasing sequence of potentials on $D$, the same inequality holds for all $u \in S^{+}(D)$. In other words, $E$ includes the half line

$$
E_{1}=\{1\} \times\left(\frac{2 \beta-1}{1-\beta},+\infty\right) .
$$

Next, by Smith-Stegenga [12, Theorem A], there is $\tau>0$ such that

$$
\iint_{D} \exp \left(\tau k_{D}(a, z)\right) d x d y<+\infty
$$

Combining (6) with (4), we obtain

$$
\iint_{D} \delta_{D}(z)^{-\tau} d x d y<+\infty
$$


or, equivalently,

$$
\iint_{D} \lambda_{D}(z)^{\tau} d x d y<+\infty,
$$

which implies that $E$ includes the half line

$$
E_{2}=\{0\} \times[-\tau,+\infty) .
$$

Now, since $E$ is a convex set by Hölder's inequality, it includes the convex hull of $E_{1} \cup E_{2}$. Consequently, there exists $p>0$, independently of $z_{0}$, for which the point $(p, 0)$ is contained in $E$. This means that $S^{+}(D) \subset L^{p}(D)$, and the proof is complete.

\section{REFERENCES}

1. D. H. Armitage, Further results on the global integrability of superharmonic functions, J. London Math. Soc. (2) 6 (1972), 109-121.

2. J. Becker and Ch. Pommerenke, Hölder continuity of conformal mappings and non-quasiconformal Jordan curves, Comment. Math. Helv. 57 (1982), 221-225.

3. F. W. Gehring and B. G. Osgood, Uniform domains and the quasi-hyperbolic metric, J. Analyse Math. 36 (1979), 50-74.

4. F. W. Gehring and B. P. Palka, Quasiconformally homogeneous domains, J. Analyse Math. 30 (1976), 172-199.

5. L. L. Helms, Introduction to potential theory, Wiley, New York, 1969.

6. I. Kra, Automorphic forms and Kleinian groups, Benjamin, Reading, MA, 1972.

7. F.-Y. Maeda and N. Suzuki, The integrability of superharmonic functions on Lipschitz domains, Bull. London Math. Soc. 21 (1989), 270-278.

8. M. Masumoto, $A$ distortion theorem for conformal mappings with an application to subharmonic functions, Hiroshima Math. J. 20 (1990), 341-350.

9. __ Integrability of superharmonic functions on plane domains, J. London Math. Soc. (2) 45 (1992), 62-78.

10. W. Smith and D. A. Stegenga, Hölder domains and Poincaré domains, Trans. Amer. Math. Soc. 319 (1990), 67-100.

11. __ Sobolev imbeddings and integrability of harmonic functions on Hölder domains, Potential Theory (Proc. Internat. Conf. Potential Theory, Nagoya, 1990) (M. Kishi, ed.), Walter de Gruyter, Berlin, 1992, pp. 303-313.

12. Exponential integrability of the quasi-hyperbolic metric on Hölder domains, Ann. Acad. Sci. Fenn. Ser. A I Math. 16 (1991), 345-360.

13. N. Suzuki, Note on the integrability of superharmonic functions, preprint.

Department of Mathematics, Hiroshima University, Hiroshima 730, Japan

Current address: Department of Mathematics, Nagoya Institute of Technology, Gokiso, Showa, Nagoya 466, Japan 